\title{
Regional observability with constraints on the state of semilinear parabolic systems
}

\author{
Hayat Zouiten ${ }^{1}$, Ali Boutoulout ${ }^{2}$ and Fatima-Zahrae El Alaoui ${ }^{3}$ \\ 1 Researcher, TSI Team, MACS Laboratory, Department of Mathematics and Computer Science, Moulay Ismail \\ University, Faculty of Sciences, 11201 Meknes, Morocco, Email: h.zouten@edu.umi.ac.ma \\ ${ }^{2}$ Full Professor, Coordinator of TSI Team $\&$ MACS Laboratory Department of Mathematics and Computer, Moulay \\ Ismail University, Faculty of Sciences, 11201 Meknes, Morocco, Email: boutouloutali@yahoo.fr \\ 3 Associate Professor, TSI Team, MACS Laboratory, Department of Mathematics and Computer Science, Moulay \\ Ismail University, Faculty of Sciences, 11201 Meknes, Morocco, Email: fzelalaoui2011@yahoo.fr
}

\begin{abstract}
The paper is devoted to the investigation of regional observability with constraints on the state of semilinear parabolic systems. The purpose is to reconstruct the initial state between two prescribed functions only on an internal subregion $\omega$ of the system evolution domain $\Omega$. The proofs use two approaches, the subdifferential and HUM approach. Finally, a numerical example is provided to verify the effectiveness of our theory results.
\end{abstract}

Keywords: Semilinear systems; Parabolic systems; Enlarged observability; Regional reconstruction; Subdifferential method; HUM approach.

Mathematics Subject Classification 2010: 35K58; 93C20; 93B07.

\section{Introduction}

Observability is one of the fundamental concept in analysis and control theory, many practical problems in various fields of engineering, physics, and chemistry are formulated as distributed systems employing PDEs and studied using this concept. Roughly speaking, observability generally means that it is possible to reconstruct the initial (state or gradient state) of a distributed system based on partial measurements takes on the system by the mean of tools called sensors.

Semilinear systems constitute an important subclass of nonlinear systems because numerous realworld problems have a semilinear structure, they include applications in nuclear, thermal, physical, chemical, etc. This kind of systems occupied now an important place in control and systems theory with a large list of publications. For example, Fabre et al. [4] prove approximate controllability in $L^{p}(\Omega)$ for $1 \leq p \leq \infty$ using a control which can be internal or on the boundary and when the nonlinearity $\mathcal{N}$ is globally Lipschitz. Moreover, in the case of the interior control, they prove approximate controllability in $\mathcal{C}_{0}(\Omega)$. Fernàndez-Cara [5] studied the null-controllability for semilinear distributed parabolic systems when the nonlinear term $\mathcal{N}$ grows slower. Zhang [16] investigated the local exact controllability of semilinear evolution systems. The fixed point theorems, and Gronwall's inequality was used by Zuazua [18] to solve the problem of controllability for semilinear systems. Zerrik et al. [15] introduced the concept of regional controllability for a class of semilinear evolution equations, they prove the exact controllability when the system is asymptotically linear. 
In the theory of distributed parameter systems, many works deal with the problem of observability for semilinear systems defined in the whole domain $\Omega$. This concept has been carried out the wide literature (see Magnusson, [11] and Baroun \& Jacob, [2]). Recently, the concept of regional observability for semilinear systems was introduced and developed by (Zerrik et al. [14] and Boutoulout et al. [3]), which they study the possibility to reconstruct the initial (state or gradient state) only on a subregion $\omega$ of the evolution domain $\Omega$.

In this paper, we study for the first time the concept of regional observability with constraints on the state for a class of semilinear parabolic systems (also called enlarged observability). The introduction of this kind of concept is motivated by many real problems. This is the case, for example, the mathematical model of real systems is obtained from measures or form approximations. Then, the solution of such systems is approximately known and required to be only between two bounds.

This paper is divided as follows. The studied problem and some preliminaries are introduced in section 2. In section 3, we characterize the enlarged observability of the system. In section 4, we give a characterization of this concept using subdifferential technics. Section 5 , is focused on the regional reconstruction of the initial state between two prescribed functions only in an internal subregion of the evolution domain. Finally, we develop a numerical approach which is illustrated by simulations that lead to some conjectures.

\section{Problem formulation and preliminaries}

Let $\Omega$ be an open subset of $\mathbb{R}^{n}(n=1,2,3)$, with a regular boundary $\partial \Omega$. For $0<T<+\infty$, let us denote $\left.Q_{T}=\Omega \times\right] 0, T\left[\right.$ and $\left.\Sigma_{T}=\partial \Omega \times\right] 0, T[$. We consider the following semilinear parabolic system:

$$
\left\{\begin{array}{llll}
\frac{\partial y}{\partial t}(x, t) & =A y(x, t)+\mathcal{N} y(x, t) & \text { in } & Q_{T} \\
y(\xi, t) & =0 & \text { on } & \Sigma_{T} \\
y(x, 0) & =y_{0}(x) & \text { in } & \Omega,
\end{array}\right.
$$

where $A$ is a second-order linear differential operator with compact resolvent which generates a strongly continuous semigroup $(S(t))_{t \geq 0}$ on a Hilbert space $X=L^{2}(\Omega)$. We suppose that $y_{0} \in L^{2}(\Omega)$ is unknown and $\mathcal{N}$ is a nonlinear continuous operator from $L^{2}(\Omega)$ to $L^{2}(\Omega)$.

We associate to the system (1) the linear one defined by

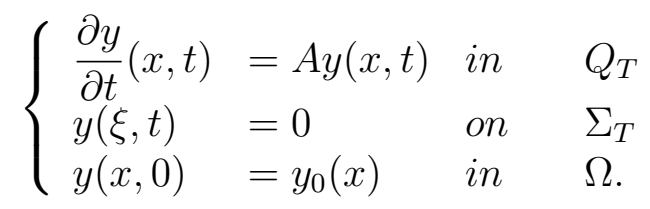

The system (2) has a unique solution $y \in L^{2}(] 0, T[\times \Omega)$ (see Lions and Magenes (1968) [8]). Without lose of generality we note $y(x, t):=y(t)$.

System (1) is augmented with the output function given by

$$
z(t)=C y(t), \quad t \in] 0, T[,
$$

where $C: D(C) \subseteq L^{2}(\Omega) \longrightarrow L^{2}\left(0, T ; \mathbb{R}^{q}\right)$, is called the observation operator is a linear operator(possibly unbounded), depends on the number $q$ and the nature of the considered sensors. The observation space is $\mathcal{O}=L^{2}\left(0, T ; \mathbb{R}^{q}\right)$. 
It is well known that global Lipschitz continuity of the nonlinear $\mathcal{N}$ implies that the problem (1) admits a unique mild solution (see Pazy (1990) [12] and Zeidler (1990) [13]) given by the variation of parameters formula

$$
y(t)=S(t) y_{0}+\int_{0}^{t} S(t-\tau) \mathcal{N} y(\tau) d \tau
$$

with $y \in L^{2}\left(0, T ; L^{2}(\Omega)\right)$.

We define a nonlinear semigroup $(F(t))_{t \geq 0}$ associated to the solution of (1) by

$$
\left.F(t) y_{0}=y(t), t \in\right] 0, T[\text {. }
$$

Hence the output function is formally given by

$$
\left.z(t)=C F(t) y_{0}, t \in\right] 0, T[,
$$

with $F(\cdot)$ is a family of nonlinear operators map $L^{2}(\Omega)$ into itself, and they satisfy the following properties:

- (P1) $F(0) x=x$, for $x \in L^{2}(\Omega)$.

- (P2) $F(t+s) x=F(t) F(s) x$, for $s, t \in] 0, T\left[\right.$ and $x \in L^{2}(\Omega)$.

- (P3) For each $x \in L^{2}(\Omega)$, the $L^{2}(\Omega)$ - valued function $F(\cdot) x$ is continuous over $[0,+\infty)$.

The first and second property is obtained through the uniqueness of mild solutions, and the third property follows from the fact that the solution $y(t)$ of (4) is continuous.

Then $(F(t))_{t \geq 0}$ is a family of nonlinear operators on $L^{2}(\Omega)$ which formed a nonlinear semigroup on $L^{2}(\Omega)$, with the above-mentioned properties (P1), (P2) and (P3). If in particular, a semigroup on $L^{2}(\Omega)$ provides mild solutions of $(1)$ in the sense of $(5)$, we call it the nonlinear semigroup on $L^{2}(\Omega)$ associated with the semilinear system (1) and we have

$$
\left.F(t) y_{0}=S(t) y_{0}+\int_{0}^{t} S(t-\tau) \mathcal{N} y(\tau) d \tau, \quad t \in\right] 0, T\left[, y_{0} \in L^{2}(\Omega) .\right.
$$

The output function is only well defined if $C$ is bounded. However, in case of unbounded observation operator, we have $C y(t)$ is not defined, then we introduce the notion of admissible operator and we have that $C$ is an admissible operator for the nonlinear semigroup $(F(t))_{t \geq 0}$ in the sense of Definition 2.1, if we can extend the mapping $y \longmapsto C F(\cdot) y$ to a bounded linear operator from $L^{2}(\Omega)$ to $L^{2}\left(0, T ; \mathbb{R}^{q}\right)$.

Definition 2.1 [2] Let $C \in \mathcal{L}(D(C), \mathcal{O})$, we say that $C$ is an admissible observation operator for $(F(t))_{t \geq 0}$, if there exists a constant $\lambda>0$ such that

$$
\left\|C F(\cdot) y_{0}-C F(\cdot) y_{0}^{\prime}\right\|_{L^{2}\left(0, T ; \mathbb{R}^{q}\right)} \leq \lambda\left\|y_{0}-y_{0}^{\prime}\right\|,
$$

for any $y_{0}, y_{0}^{\prime} \in D(C)$.

Let us consider the observability operators defined by

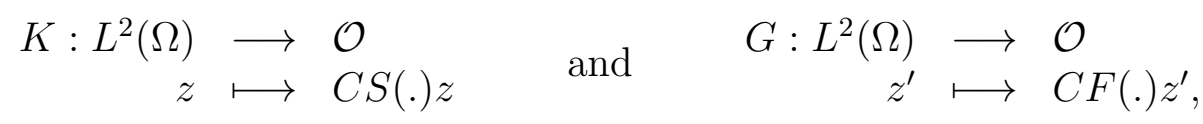

their adjoints $K^{*}$ and $G^{*}$ are given by

$$
\begin{aligned}
K^{*}: \mathcal{O} & \longrightarrow L^{2}(\Omega) & G^{*}: \mathcal{O} & \longrightarrow L^{2}(\Omega) \\
z & \longmapsto \int_{0}^{T} S^{*}(t) C^{*} z(t) d t \quad \text { and } & & z^{\prime} \longmapsto \int_{0}^{T} F^{*}(t) C^{*} z^{\prime}(t) d t .
\end{aligned}
$$




\section{Regional enlarge observability}

For $\omega$ be an intern subregion of $\Omega$ with a positive Lebesgue measure. We consider the restriction operator

$$
\begin{aligned}
\chi_{\omega}: L^{2}(\Omega) & \longrightarrow L^{2}(\omega) \\
y & \longmapsto \chi_{\omega} y=y_{\mid \omega} .
\end{aligned}
$$

Its adjoint $\chi_{\omega}^{*}$ is given by

$$
\chi_{\omega}^{*} y:=\left\{\begin{array}{lll}
y & \text { in } & \omega \\
0 & \text { in } & \Omega \backslash \omega
\end{array}\right.
$$

Let $\alpha(\cdot)$ and $\beta(\cdot)$ be two functions defined in $L^{2}(\omega)$ such that $\alpha(\cdot) \leq \beta(\cdot)$ a.e. in $\omega$. Throughout the paper we set

$$
[\alpha(\cdot), \beta(\cdot)]=\left\{y \in L^{2}(\omega) \mid \alpha(\cdot) \leq y(\cdot) \leq \beta(\cdot) \text { a.e. in } \omega\right\}
$$

Definition 3.1 1. We say that the linear system (2) together with the output (3) is exactly $[\alpha(\cdot), \beta(\cdot)]$-observable in $\omega$ if

$$
\operatorname{Im}\left(\chi_{\omega} K^{*}\right) \cap[\alpha(\cdot), \beta(\cdot)] \neq \emptyset
$$

2. We say that the system (1) together with the output (3) is exactly $[\alpha(\cdot), \beta(\cdot)]$-observable in $\omega$ if

$$
\operatorname{Im}\left(\chi_{\omega} G^{*}\right) \cap[\alpha(\cdot), \beta(\cdot)] \neq \emptyset
$$

Remark 3.1 If the system (1) together with the output (3) is exactly $[\alpha(\cdot), \beta(\cdot)]$-observable in $\omega_{1}$ then it is exactly $[\alpha(\cdot), \beta(\cdot)]$-observable in any subregion $\omega_{2} \subseteq \omega_{1}$.

The study of Regional Enlarged Observability (REO) of a distributed parameter system governed by semilinear parabolic equations amounts to solve the following problem.

\section{Problem.}

Given the system (1) together with the output (3) in $\omega$ at time $t \in[0, T]$, is it possible to reconstruct $y(0)_{\left.\right|_{\omega}}=\chi_{\omega} y_{0}$ between $\alpha(\cdot)$ and $\beta(\cdot)$ in $\omega$ ?

\section{Subdifferential approach}

The purpose of this section is to apply the subdifferential approach (see Aubin (1984) [1]) to solve the problem of regional enlarged observability of semilinear parabolic system observed by internal zonal sensor and reconstruct the initial state $y_{0}$ between $\alpha(\cdot)$ and $\beta(\cdot)$ in $\omega$.

This problem is equivalent to minimize the reconstruction error given by

$$
\left\{\begin{array}{l}
\min \|G y-z\|_{\mathcal{O}}^{2} \\
y \in Y,
\end{array}\right.
$$

where $Y=\left\{y \in L^{2}(\Omega) \mid \chi_{\omega} y \in[\alpha(\cdot), \beta(\cdot)]\right\}$.

In this section, we are interested to solve the problem of minimization (10). 
Let us denote by:

- $\Gamma_{0}\left(L^{2}(\Omega)\right)$ the set of functions $\left.\left.f: L^{2}(\Omega) \longrightarrow \tilde{\mathbb{R}}=\right]-\infty,+\infty\right]$ which are proper, lower semicontinuous (l.s.c) and convex in $L^{2}(\Omega)$.

- For $f \in \Gamma_{0}\left(L^{2}(\Omega)\right)$, the domain of $f$ is defined by

$$
\operatorname{dom}(f)=\left\{y \in L^{2}(\Omega) \mid f(y)<\infty\right\} .
$$

- For $y^{0} \in \operatorname{dom}(f)$, the subdifferential of $f$ at $y^{0}$ is given by the set

$$
\partial f\left(y^{0}\right)=\left\{y^{*} \in L^{2}(\Omega) \mid f(y) \geq f\left(y^{0}\right)+\left\langle y^{*}, y-y^{0}\right\rangle, \forall y \in L^{2}(\Omega)\right\},
$$

- For $\mathcal{J}$ a nonempty subset of $L^{2}(\Omega)$

$$
\Psi_{\mathcal{J}}(y)= \begin{cases}0 & \text { if } y \in \mathcal{J} \\ +\infty & \text { otherwise }\end{cases}
$$

denotes the indicator function of $\mathcal{J}$.

With all these notations, the problem 10 is equivalent to the following problem:

$$
\left\{\begin{array}{l}
\inf \left(\|G y-z\|_{\mathcal{O}}^{2}+\Psi_{Y}(y)\right) \\
y \in L^{2}(\Omega)
\end{array}\right.
$$

Hence the solution of (11) is characterized by the following result.

Theorem 4.1 If the system (1) together with the output (3) is exactly $[\alpha(\cdot), \beta(\cdot)]$-observable in $\omega$, then the following assertions are equivalent.

1. $y^{*}$ is a solution of (11).

2. $y^{*} \in Y$ and $\Psi_{Y}^{*}\left(-2 G^{*}\left(G y^{*}-z\right)\right)=-2\left\|G y^{*}\right\|_{\mathcal{O}}^{2}+2\left\langle G^{*} z, y^{*}\right\rangle$.

\section{Proof.}

Let us consider $f(y)=\|G y-z\|_{\mathcal{O}}^{2}$, we have $y^{*}$ solution of 11 if and only if

$$
0 \in \partial\left(f+\Psi_{Y}\right)\left(y^{*}\right) \text { (Fermat's rule). }
$$

It is clear that $f \in \Gamma_{0}\left(L^{2}(\Omega)\right)$ and since $Y$ is closed, convex and nonempty, we obtain $\Psi_{Y} \in \Gamma_{0}\left(L^{2}(\Omega)\right)$.

Moreover, under the hypothesis that the system (1) together with the output (3) is exactly $[\alpha(\cdot), \beta(\cdot)]$-observable in $\omega$, we have $\operatorname{dom}(f) \cap \operatorname{dom}\left(\Psi_{Y}\right) \neq \emptyset$. Since $f$ is continuous we obtain

$$
\partial\left(f+\Psi_{Y}\right)\left(y^{*}\right)=\partial f\left(y^{*}\right)+\partial \Psi_{Y}\left(y^{*}\right)
$$

it follows that $y^{*}$ is a solution of (11) if and only if $0 \in \partial f\left(y^{*}\right)+\partial \Psi_{Y}\left(y^{*}\right)$.

Moreover, $f$ is Frechet-differentiable, then

$$
\partial f\left(y^{*}\right)=\left\{\nabla f\left(y^{*}\right)\right\}=\left\{2 G^{*}\left(G y^{*}-z\right)\right\},
$$

thus $y^{*}$ is a solution of $(11)$ if and only if

$$
-2 G^{*}\left(G y^{*}-z\right) \in \partial \Psi_{Y}\left(y^{*}\right),
$$

which is equivalent to

$$
y^{*} \in Y, \quad \Psi_{Y}^{*}\left(-2 G^{*}\left(G y^{*}-z\right)\right)=-2\left\|G y^{*}\right\|_{\mathcal{O}}^{2}+2\left\langle G^{*} z, y^{*}\right\rangle .
$$

The proof is complete.

Remark 4.1 This approach is difficult to implement numerically, that is why we are exposing in the next paragraph an approach which can be implemented by numerical examples. 


\section{$5 \quad$ HUM approach}

The purpose of this paragraph is to give an extension of the Hilbert Uniqueness Method introduced in the linear case by Lions (see Lions (1988) [9], (1989) [10]), which allows the determination of the initial state between $\alpha(\cdot)$ and $\beta(\cdot)$ in $\omega$ and does not take into account the residual part between $\alpha(\cdot)$ and $\beta(\cdot)$ in the subregion $\Omega \backslash \omega$.

Let the initial state decomposed in the following form:

$$
y_{0}=\left\{\begin{array}{lll}
y_{0}^{1} & \text { in } & {[\alpha(\cdot), \beta(\cdot)]} \\
y_{0}^{2} & \text { in } & L^{2}(\Omega) \backslash[\alpha(\cdot), \beta(\cdot)] .
\end{array}\right.
$$

In this section, our subject is the reconstruction of the component $y_{0}^{1}$. We consider the system (1) observed by an internal zonal sensor $(D, f)$. where $D$ is a nonempty closed part of $\bar{\Omega}$ representing the geometric support of the sensor, and $f$ define the spatial distribution of the information on the support $D$, for more details (see [6], [7]). Then the output function is given by

$$
z(t)=\int_{D} y(x, t) f(x) d x .
$$

Let $\mathcal{G}$ be defined by

$$
\mathcal{G}=\left\{g \in L^{2}(\Omega) \mid g=0 \text { in } L^{2}(\Omega) \backslash[\alpha(\cdot), \beta(\cdot)]\right\} .
$$

We consider the semilinear system given by

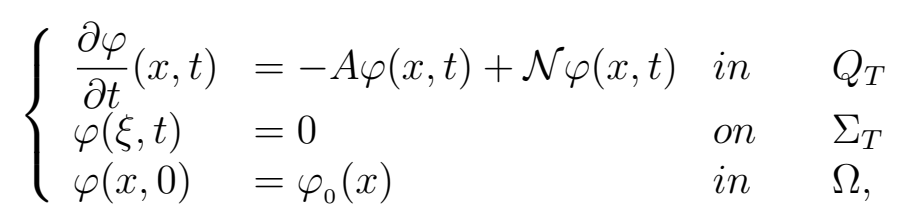

which can be decomposed as the follows:

$$
\left\{\begin{array}{llll}
\frac{\partial \varphi_{1}}{\partial t}(x, t) & =-A \varphi_{1}(x, t) & \text { in } & Q_{T} \\
\varphi_{1}(\xi, t) & =0 & \text { on } & \Sigma_{T} \\
\varphi_{1}(x, 0) & =\varphi_{0}(x) & \text { in } & \Omega
\end{array}\right.
$$

and

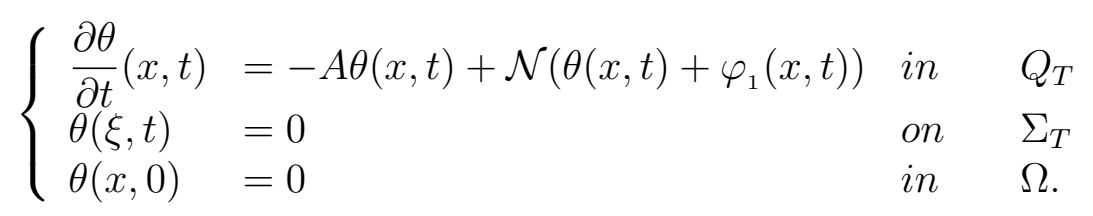

Without loss of generality, we note $\varphi_{1}(x, t):=\varphi_{1}(t)$ and $\theta(x, t):=\theta(t)$.

Let us consider $\varphi_{0} \in \mathcal{G}$, we define the mapping

$$
\varphi_{0} \longmapsto\left\|\varphi_{0}\right\|_{\mathcal{G}}=\left(\int_{0}^{T}\left\langle\varphi_{1}(t), f\right\rangle_{L^{2}(D)}^{2} d t\right)^{\frac{1}{2}},
$$

which induces a semi-norm in $\mathcal{G}$. If the linear system $[16$ is exactly $[\alpha(\cdot), \beta(\cdot)]$-observable in $\omega$, then the semi-norm defines a norm in $\mathcal{G}$ (see Zouiten et al. (2017) [17]), we also designate by $\mathcal{G}$ 
the completion of $\mathcal{G}$ with respect to the norm $(18)$.

We define the auxiliary system by

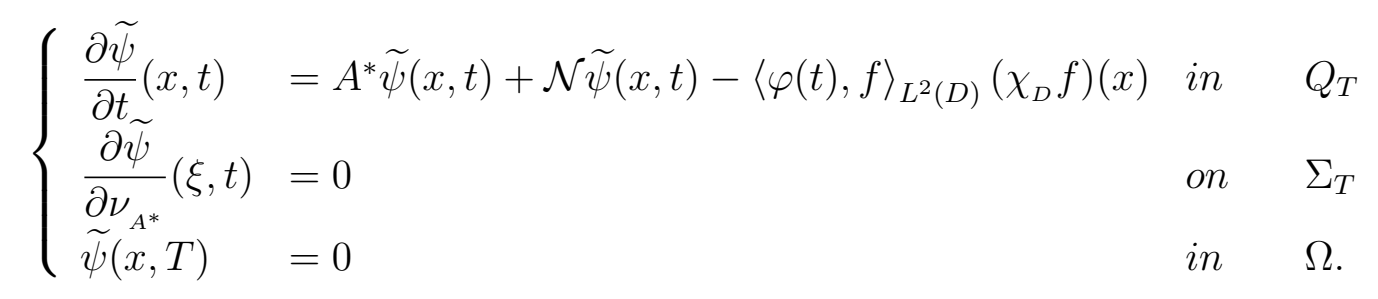

Consider the nonlinear operator $\mu$ defined by

$$
\begin{aligned}
\mu: \mathcal{G} & \longrightarrow \mathcal{G}^{*} \\
\varphi_{0} & \longmapsto \mathcal{P}(\widetilde{\psi}(0)),
\end{aligned}
$$

where $\mathcal{P}=\chi_{\omega}^{*} \chi_{\omega}$ the projection operator.

We consider the following decomposition $\widetilde{\psi}=\psi_{0}+\psi_{1}$, where $\psi_{0}$ and $\psi_{1}$ are the solutions of the following systems:

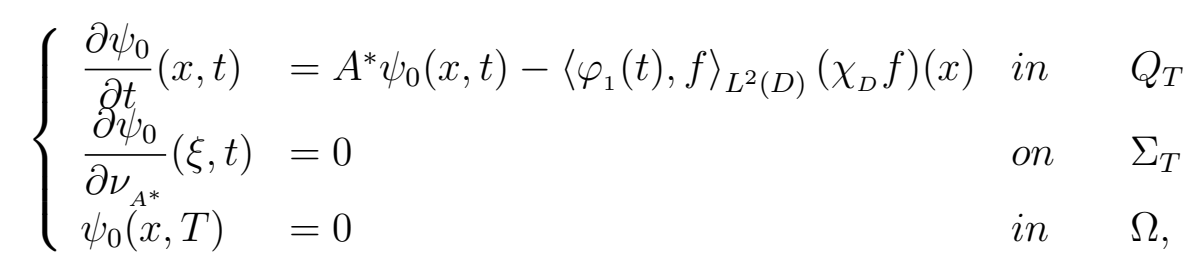

and

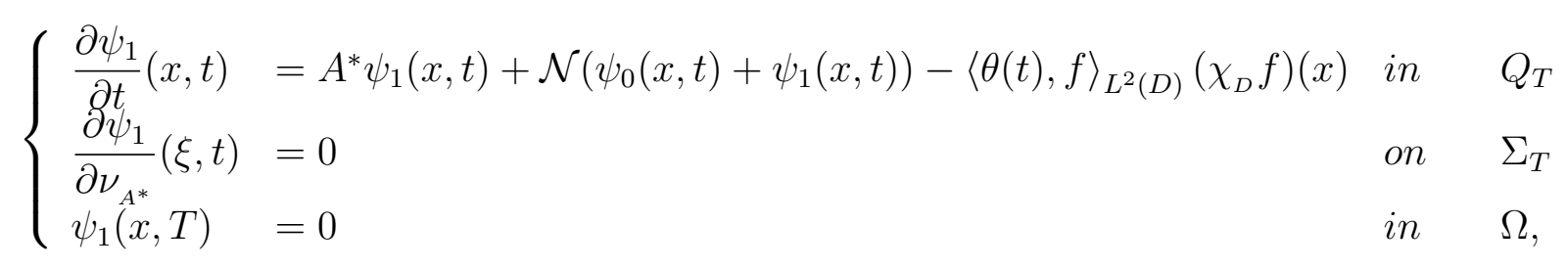

we have

$$
\mu \varphi_{0}=\mathcal{P}\left(\psi_{0}(0)\right)+\mathcal{P}\left(\psi_{1}(0)\right)
$$

We consider the following operators:

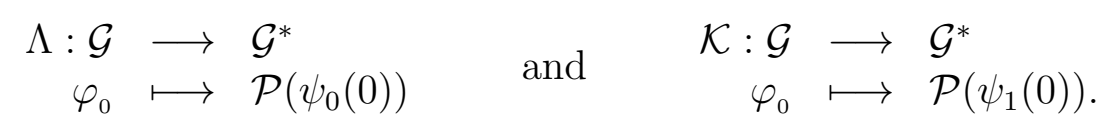

Then

$$
\mu\left(\varphi_{0}\right)=\underbrace{\Lambda\left(\varphi_{0}\right)}_{\text {linear part }}+\underbrace{\mathcal{K}\left(\varphi_{0}\right)}_{\text {nonlinear part }} .
$$

If the linear system 16$]$ is exactly $[\alpha(\cdot), \beta(\cdot)]$-observable in $\omega$, then $\Lambda$ is an isomorphism (see [17]), and we obtain

$$
\varphi_{0}=\Lambda^{-1} \mathcal{P}(\widetilde{\psi}(0))-\Lambda^{-1} \mathcal{K} \varphi_{0}
$$


Considering the following system:

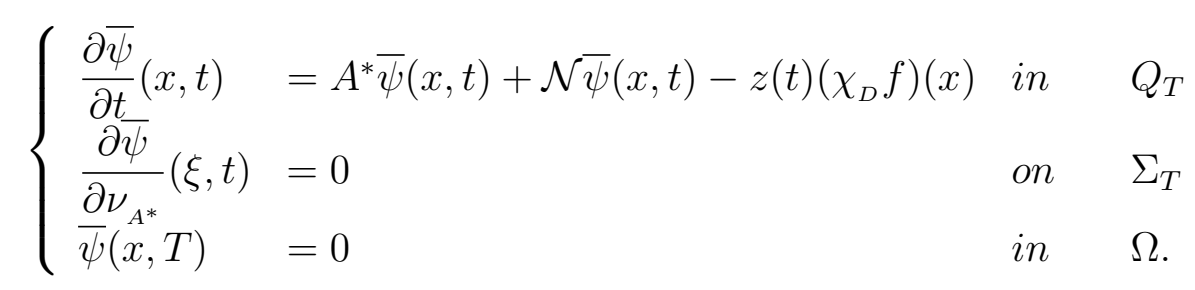

If $\varphi_{0}$ is chosen such that $\bar{\psi}(0)=\widetilde{\psi}(0)$ in $\omega$, then the system 23 can be seen like the adjoint of the system (1) and our problem of regional enlarged observability turns up to find the fixed point of the equation

$$
\Phi\left(\varphi_{0}\right)=\varphi_{0}
$$

where

$$
\begin{aligned}
\Phi: \mathcal{G} & \longrightarrow \mathcal{G}^{*} \\
\varphi_{0} & \longrightarrow \Lambda^{-1} \mathcal{P}(\bar{\psi}(0))-\Lambda^{-1} \mathcal{K} \varphi_{0}
\end{aligned}
$$

then we have the following result.

Theorem 5.1 If the linear system (2) together with the output (3) is exactly $[\alpha(\cdot), \beta(\cdot)]$-observable in $\omega$, and $\mathcal{N}$ satisfies that there exists $\delta>0$ such that

$$
\|\mathcal{N}(x)\| \leq \delta\|x\|,
$$

then the equation (24) admits a unique fixed point $\varphi_{0}$ corresponding to the initial state to be observed between $\alpha(\cdot)$ and $\beta(\cdot)$ in $\omega$.

\section{Proof.}

We have

$$
\Phi\left(\varphi_{0}\right)=\Lambda^{-1} \mathcal{P}(\bar{\psi}(0))-\Lambda^{-1} \mathcal{K} \varphi_{0},
$$

we show under some hypothesis that the operator $\Phi$ has a unique fixed point which corresponds to the initial state to be observed between $\alpha(\cdot)$ and $\beta(\cdot)$ in $\omega$ via two steps.

Step 1. Let us consider $p>0$, and $\mathcal{B}_{P}=\mathcal{B}(0, p)$, we have

$$
\mathcal{H}\left(\mathcal{B}_{P}\right)=\left\{\mathcal{P}\left(\psi_{1}(0)\right) \mid \varphi_{0} \in \mathcal{B}_{p}\right\}
$$

and

$$
\widetilde{\mathcal{B}}_{P}=\left\{\mathcal{P}\left(\psi_{1}(t)\right) \mid \varphi_{0} \in \mathcal{B}_{p}, t \in[0, T]\right\} .
$$

We find that $\mathcal{H}\left(\mathcal{B}_{P}\right) \subset \widetilde{\mathcal{B}}_{P}$, then it is sufficient to show that $\widetilde{\mathcal{B}}_{P}$ is relatively compact.

We have $\psi_{1}(\cdot)$ is a solution of (21), then

$$
\psi_{1}(t)=S(t) \psi_{1}(T)+\int_{T}^{t} S(t-\tau)\left[\mathcal{N}\left(\psi_{0}(\tau)+\psi_{1}(\tau)\right)-\langle\theta(\tau), f\rangle\left(\chi_{D} f\right)(x)\right] d \tau
$$

we obtain

$$
\psi_{1}(t)=-\int_{t}^{T} S(t-\tau)\left[\mathcal{N}\left(\psi_{0}(\tau)+\psi_{1}(\tau)\right)-\langle\theta(\tau), f\rangle\left(\chi_{D} f\right)(x)\right] d \tau
$$


with $\psi_{1}(\cdot) \in C\left(0, T ; L^{2}(\Omega)\right)$. Then

$$
\exists \eta>0 \text { such that }\left\|\mathcal{P}\left(\psi_{1}(t)\right)\right\|_{\mathcal{G}^{*}} \leq \eta\left\|\psi_{1}(t)\right\| .
$$

Since $(S(t))_{t \geq 0}$ is a strongly continuous semigroup in $[0, T]$, we have

$$
\exists M>0 \text { such that }\|S(t)\| \leq M, \quad \forall t \in[0, T] .
$$

According to the expression of $\psi_{1}(t)$, we have

$$
\left\|\psi_{1}(t)\right\| \leq M \int_{t}^{T}\left[\delta\left(\left\|\psi_{0}(\tau)\right\|+\left\|\psi_{1}(\tau)\right\|\right)+\|\theta(\tau)\|\|f\|^{2}\right] d \tau .
$$

Since $\psi_{0}(\cdot)$ is a solution of 20 , then

$$
\psi_{0}(t)=-\int_{T}^{t} S(t-\tau)\left\langle\varphi_{1}(\tau), f\right\rangle\left(\chi_{D} f\right)(x) d \tau,
$$

we obtain

$$
\left\|\psi_{0}(t)\right\| \leq M \int_{t}^{T}\left\|\varphi_{1}(\tau)\right\|\|f\|^{2} d \tau
$$

Or $\varphi_{1}$ is a solution of (16), thus

$$
\begin{gathered}
\varphi_{1}(t)=-S(t) \varphi_{0}, \\
\left\|\varphi_{1}(t)\right\| \leq M\left\|\varphi_{0}\right\| .
\end{gathered}
$$

Then

$$
\int_{t}^{T}\left\|\varphi_{1}(\tau)\right\| d \tau \leq T M\left\|\varphi_{0}\right\|
$$

Hence

$$
\left\|\psi_{0}(t)\right\| \leq T M^{2}\|f\|^{2}\left\|\varphi_{0}\right\|
$$

we obtain

$$
\int_{t}^{T}\left\|\psi_{0}(\tau)\right\| d \tau \leq T^{2} M^{2}\|f\|^{2}\left\|\varphi_{0}\right\| .
$$

On the other hand, we have $\theta(\cdot)$ is a solution of (17), then

$$
\theta(t)=\int_{0}^{t} S(t-\tau) \mathcal{N}\left[\theta(\tau)+\varphi_{1}(\tau)\right] d \tau,
$$

which give

$$
\|\theta(t)\| \leq M \delta \int_{0}^{t}\|\theta(\tau)\|+\left\|\varphi_{1}(\tau)\right\| d \tau
$$

therefore

$$
\|\theta(t)\| \leq t M^{2} \delta\left\|\varphi_{0}\right\|+M \delta \int_{0}^{t}\|\theta(\tau)\| d \tau .
$$

Since $t \longmapsto t M^{2} \delta\left\|\varphi_{0}\right\|$ is nondecreasing and using Gronwall theorem, we obtain

$$
\|\theta(t)\| \leq T M^{2} \delta e^{T M \delta}\left\|\varphi_{0}\right\|
$$


then

$$
\int_{t}^{T}\|\theta(\tau)\| d \tau \leq T^{2} M^{2} \delta e^{T M \delta}\left\|\varphi_{0}\right\| .
$$

Finally, we obtain

$$
\left\|\psi_{1}(t)\right\| \leq T^{2} M^{3} \delta\|f\|^{2}\left[1+e^{T M \delta}\right]\left\|\varphi_{0}\right\|+M \delta \int_{t}^{T}\left\|\psi_{1}(\tau)\right\| d \tau
$$

by Gronwall theorem

$$
\left\|\psi_{1}(t)\right\| \leq e^{T M \delta}\left(T^{2} M^{3} \delta\|f\|^{2}\left[1+e^{T M \delta}\right]\right)\left\|\varphi_{0}\right\|
$$

Then $\widetilde{\mathcal{B}}_{p}$ is uniformly bounded.

Now let us show that $\widetilde{\mathcal{B}}_{p}$ is equicontinuous. Indeed, we have

$$
\begin{aligned}
\psi_{1}\left(t_{2}\right)-\psi_{1}\left(t_{1}\right) & =\int_{T}^{t_{2}} S\left(t_{2}-\tau\right)\left[\mathcal{N}\left(\psi_{0}(\tau)+\psi_{1}(\tau)\right)-\langle\theta(\tau), f\rangle\left(\chi_{D} f\right)(x)\right] d \tau \\
& -\int_{T}^{t_{1}} S\left(t_{1}-\tau\right)\left[\mathcal{N}\left(\psi_{0}(\tau)+\psi_{1}(\tau)\right)-\langle\theta(\tau), f\rangle\left(\chi_{D} f\right)(x)\right] d \tau
\end{aligned}
$$

we obtain

$$
\begin{aligned}
\psi_{1}\left(t_{2}\right)-\psi_{1}\left(t_{1}\right) & =\int_{T}^{t_{2}}\left(S\left(t_{2}-\tau\right)-S\left(t_{1}-\tau\right)\right)\left[\mathcal{N}\left(\psi_{0}(\tau)+\psi_{1}(\tau)\right)-\langle\theta(\tau), f\rangle\left(\chi_{D} f\right)(x)\right] d \tau \\
& +\int_{t_{1}}^{t_{2}} S\left(t_{1}-\tau\right)\left[\mathcal{N}\left(\psi_{0}(\tau)+\psi_{1}(\tau)\right)-\langle\theta(\tau), f\rangle\left(\chi_{D} f\right)(x)\right] d \tau \\
& =\mathcal{D}_{1}+\mathcal{D}_{2},
\end{aligned}
$$

where

$$
\begin{aligned}
& \mathcal{D}_{1}=\int_{T}^{t_{2}}\left(S\left(t_{2}-\tau\right)-S\left(t_{1}-\tau\right)\right)\left[\mathcal{N}\left(\psi_{0}(\tau)+\psi_{1}(\tau)\right)-\langle\theta(\tau), f\rangle\left(\chi_{D} f\right)(x)\right] d \tau \\
& \mathcal{D}_{2}=\int_{t_{1}}^{t_{2}} S\left(t_{1}-\tau\right)\left[\mathcal{N}\left(\psi_{0}(\tau)+\psi_{1}(\tau)\right)-\langle\theta(\tau), f\rangle\left(\chi_{D} f\right)(x)\right] d \tau
\end{aligned}
$$

We obtain that for all $\varepsilon_{1}>0$, there exists $\zeta>0$ such that $\left|t_{2}-t_{1}\right|<\zeta$ implies

$$
\left\|S\left(t_{2}-\tau\right)-S\left(t_{1}-\tau\right)\right\| \leq \varepsilon_{1} \quad \forall t \in[0, T],
$$

which yields

$$
\left\|\mathcal{D}_{1}\right\| \leq \varepsilon_{1} T^{2} M^{2} \delta\|f\|^{2}\left(\left[1+T M \delta e^{T M \delta}\right]\left[1+e^{T M \delta}\right]\right)\left\|\varphi_{0}\right\|
$$

and

$$
\left\|\mathcal{D}_{2}\right\| \leq \zeta T^{2} M^{3} \delta\|f\|^{2}\left(\left[1+T M \delta e^{T M \delta}\right]\left[1+e^{T M \delta}\right]\right)\left\|\varphi_{0}\right\| .
$$

Then

$$
\begin{aligned}
\left\|\mathcal{P}\left(\psi_{1}\left(t_{2}\right)\right)-\mathcal{P}\left(\psi_{1}\left(t_{1}\right)\right)\right\|_{\mathcal{G}^{*}} & \leq \eta\left\|\psi_{1}\left(t_{2}\right)-\psi_{1}\left(t_{1}\right)\right\| \\
& \leq \varepsilon_{1} \eta \mathcal{F}_{1}+\zeta \eta \mathcal{F}_{2},
\end{aligned}
$$


with

$$
\left\{\begin{array}{l}
\mathcal{F}_{1}=T^{2} M^{2} \delta\|f\|^{2}\left(\left[1+T M \delta e^{T M \delta}\right]\left[1+e^{T M \delta}\right]\right)\left\|\varphi_{0}\right\|, \\
\mathcal{F}_{2}=T^{2} M^{3} \delta\|f\|^{2}\left(\left[1+T M \delta e^{T M \delta}\right]\left[1+e^{T M \delta}\right]\right)\left\|\varphi_{0}\right\| .
\end{array}\right.
$$

For

$$
\varepsilon_{1} \leq \frac{\varepsilon}{2 \eta \mathcal{F}_{1}} \quad \text { and } \quad \zeta \leq \frac{\varepsilon}{2 \eta \mathcal{F}_{2}}
$$

we obtain

$$
\left\|\mathcal{P}\left(\psi_{1}\left(t_{2}\right)\right)-\mathcal{P}\left(\psi_{1}\left(t_{1}\right)\right)\right\|_{\mathcal{G}^{*}} \leq \varepsilon
$$

Thus, the operator $\Phi: \mathcal{B}_{p} \longrightarrow \mathcal{G}^{*}$ is compact.

Step 2. $\Phi$ enforces the ball $\mathcal{B}_{p}$ in itself. If the system (2) is exactly $[\alpha(\cdot), \beta(\cdot)]$-observable in $\omega$, then $\Lambda^{-1} \mathcal{P}$ is bounded and we have

$$
\left\|\Phi\left(\varphi_{0}\right)\right\| \leq\left\|\Lambda^{-1} \mathcal{P}\right\|\left(\|\bar{\psi}(0)\|+\left\|\psi_{1}(0)\right\|\right) .
$$

Then applying the theorem of Schauder (see Zeidler (1990) [13]) the operator $\Phi$ admits a unique fixed point and the initial state to be observed between $\alpha(\cdot)$ and $\beta(\cdot)$ in $\omega$ is given by

$$
y_{0}^{1}=\chi_{\omega} \varphi_{0}
$$

The proof is complete.

Remark 5.1 One can proceed in the same way if the system (1) is augmented with

$$
z(t)=y(b, t), \quad b \in \Omega, t \in] 0, T[
$$

which is observed by a pointwise sensor.

Assume that the system (2) is exactly $[\alpha(\cdot), \beta(\cdot)]$-observable in $\omega$. Then we have the following algorithm.

\section{Algorithm.}

Step 1 : • The subregion $\omega$, the domain $D$ and the function of distribution $f$.

- Choose the function $y_{0} \in[\alpha(\cdot), \beta(\cdot)]$.

- Threshold accuracy $\varepsilon$.

Step 2 : Repeat
$\triangleright$ Solve the system $(16)$ to obtain $\varphi_{1}$.
$\triangleright$ Solve the system $(\overline{17})$ to obtain $\theta$.
$\triangleright$ Solve the system $(20)$ to obtain $\psi_{0}$.
$\triangleright$ Solve the system $(\overline{21})$ to obtain $\psi_{1}$.
$\triangleright$ Resolution of $\Phi\left(\varphi_{0}\right)=\varphi_{0}$, and obtain $\varphi_{0}$.
Until $\left\|\varphi_{0}-\Phi\left(\varphi_{0}\right)\right\|<\varepsilon$.

Step 3 : The solution $\varphi_{0}$ corresponds to the initial state to be observed between $\alpha(\cdot)$ and $\beta(\cdot)$ in $\omega$. 


\section{Simulation results}

Here we present a numerical example that leads to some results related to the choice of the subregion, the constraints functions, and the sensor location.

Let us consider the system defined for $\Omega=] 0,1[$ by the following one-dimensional system observed by a pointwise sensor:

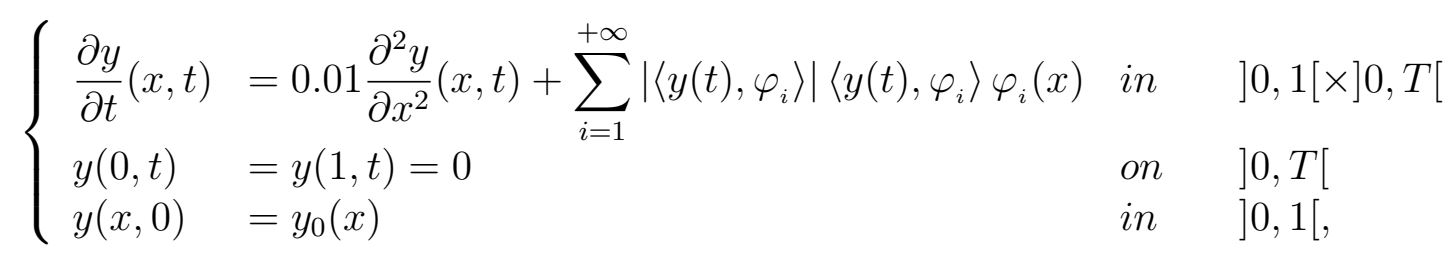

where the operator $A=\frac{\partial^{2}}{\partial x^{2}}$ has a complete set of eigenfunctions $\left(\varphi_{i}\right)_{i \in \mathbb{N}}$ in $L^{2}(\Omega)$.

The system (25) is augmented with the output function given by

$$
z(t)=y(b, t), \quad t \in] 0, T[
$$

with $b=0.83$ and $T=2$. Let us consider

$$
\begin{aligned}
y_{0}(x) & =(x-1)^{2} \sin ^{2}(x), \\
\alpha(x) & =\frac{2}{11} x(1-x), \\
\beta(x) & =\frac{1}{4} x(1-x) .
\end{aligned}
$$

Applying the previous algorithm, we obtain the following results.

- For $\omega=] 0.25,0.70[$

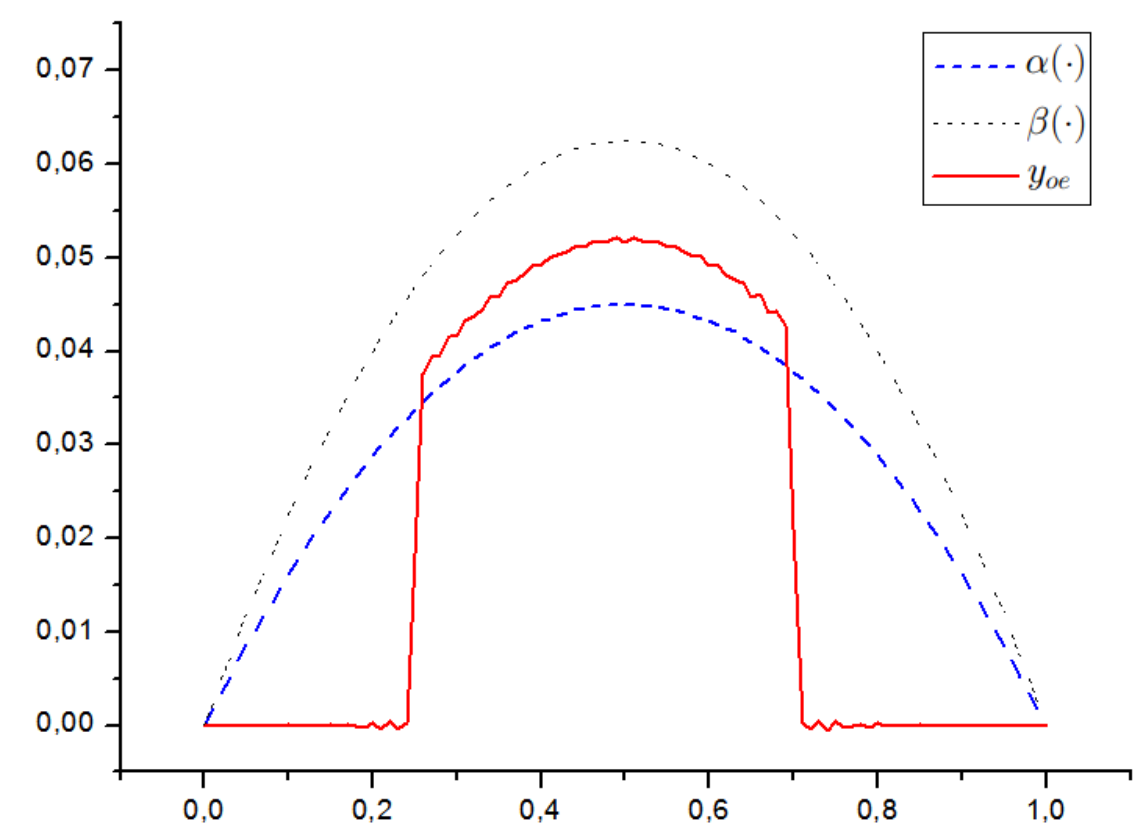

Figure 1 - The estimated initial state $y_{0 e}$ 
Figure. 1 shows that the initial state estimated $y_{o e}$ is between $\alpha(\cdot)$ and $\beta(\cdot)$ in $\left.\omega=\right] 0.25,0.70[$, then the system $(25)-(26)$ is $[\alpha(\cdot), \beta(\cdot)]$-observable in $\omega$.

The initial state $y_{o e}$ is estimated with a reconstruction error

$$
\left\|y_{0}-y_{o e}\right\|^{2}=1.8 \times 10^{-3}
$$

- If the sensor is located in $b=0.78$, we obtain

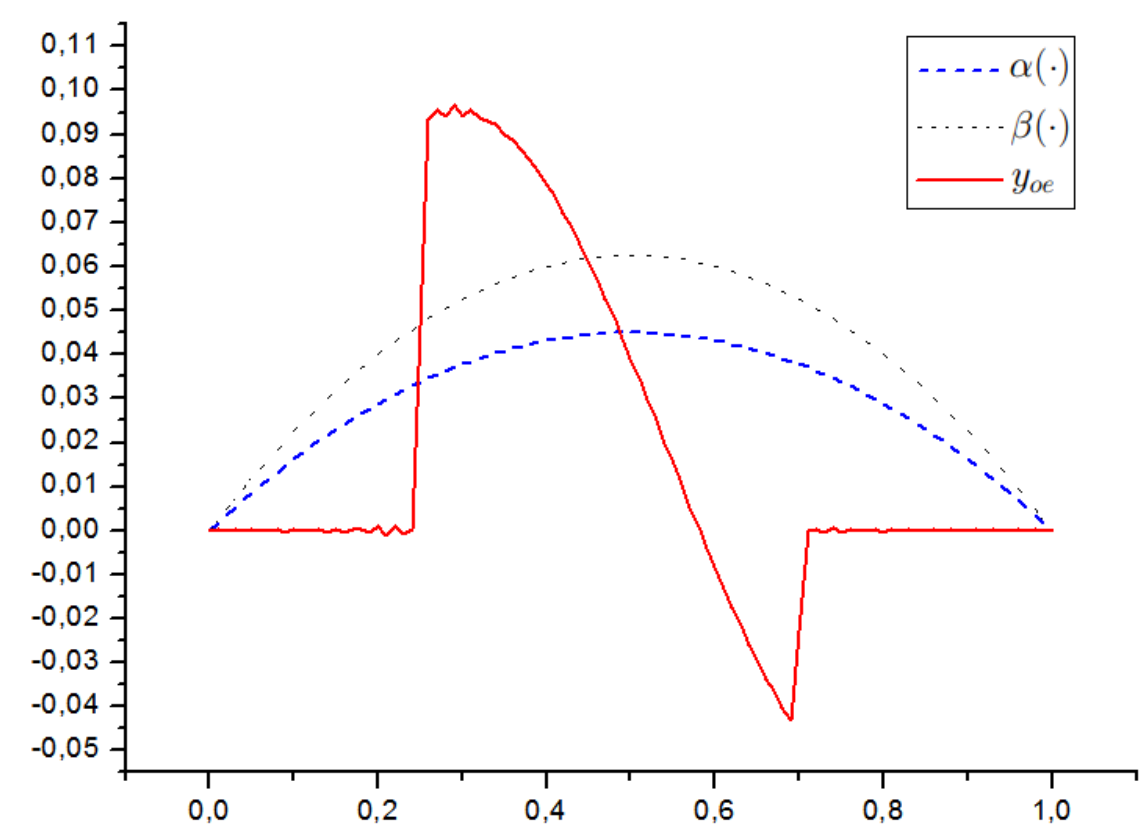

Figure 2 - The estimated initial state $y_{0 e}$

Figure. 2 shows that the initial state estimated $y_{o e}$ is not between $\alpha(\cdot)$ and $\beta(\cdot)$ in $\omega=] 0.25,0.70[$, this means that the system $(25)-26)$ is not $[\alpha(\cdot), \beta(\cdot)]$-observable in $\omega$.

\section{Conclusion}

This paper deals with the notion of regional enlarged observability of semilinear parabolic systems. The internal enlarged observability of linear systems was used to solve this problem. Interesting results are obtained and illustrated with numerical example and simulations. Many questions remain open, such as the case of the regional enlarged observability of semilinear systems using Lagrangian approach and the case of the regional enlarged observability for a class of fractional order systems. Those questions are still under consideration and the results will appear in a future paper.

\section{Acknowledgments}

This work has been carried out with a grant from Hassan II Academy of Sciences and Technology project $630 / 2016$. 


\section{References}

[1] J.P. Aubin (1984), L'analyse non linéaire et ses motivations economiques, Dunod.

[2] M. Baroun And B. JaCOB (2009), Admissibility and observability of observation operators for semilinear problems, Integral Equations Operator Theory, 64, 1-20.

[3] A. Boutoulout, H. Bourray And E.Z. El Alaoui (2010), Some extension of the regional observability for distributed semilinear parabolic systems : theory and simulation, Int. Journal of Math. Analysis, 4(24), 1153-1173.

[4] C. Fabre, P.J. Puel and E. Zuazua (1995), Approximate controllability of the semilinear heat equation, Proc. Roy Soc. Edinburgh 125 A, 31-61.

[5] E. Fernàndez- Cara And E. Zuazua (2000), Null and approximate controllability for weakly blowing up semilinear heat equation, Annales de L'Institut Henri Poincaré, Non Linear Analysis, 17 (5), 583-616.

[6] A. EL Jai, M.C Simon And E. ZerRIK (1993), Regional observability and sensors structures, Sensors and Actuators Journal, Vol 39, 95-102.

[7] A. EL Jai And A.J. Pritchard (1988), Sencors and actuators in distributed systeme analysis, Ellis Horwood Series in Applied Mathematics, J. Wiley.

[8] J.L. Lions And E. Magenes (1968), Problèmes aux limites non homogènes et applications, Vol 1 et 2, Dunod, Paris.

[9] J.L. Lions (1988), Contrôlabilité exacte perturbations et stabilisation des systèmes distribués, Tome 1, Contrôlabilité Exacte, Masson, Paris.

[10] J.L. Lions (1989), Sur la contrôlabilité exacte élargie, Progress in Nonlinear Differential Equations and Their Applications, Vol 1, 703-727.

[11] K. G. Magnusson (1984), Observability of nonlinear systems, IMA J. Math. Control Information 1, 339-358.

[12] A. PAZY (1990), Semigroups of linear operators and applications to partial differential equations, New York: Springer.

[13] E. ZEIDLER (1990), Nonlinear functional analysis and its applications II/A linear applied functional analysis, Berlin: Springer.

[14] E. Zerrik, H. Bourray And A. El Jai (2004), Regional observability for semilinear distributed parabolic systems, Journal of Dynamical and Control Systems, Vol. 10, No. 3, 413-430.

[15] E. Zerrik, R. LARHrissi And H. Bourray (2007), An output controllability problem for semilinear distributed hyperbolic systems, Int. J. Appl. Math. Comput. Sci, 17 4, 437-448.

[16] X. Zhang (2000), Exact controllability of semilinear evolution systems and its application, Journal of Optimization Theory and Applications, 107, No. 2, 415-432. 
[17] H. Zouiten, A. Boutoulout and F.Z. El Alaoui (2017), On the regional enlarged observability for linear parabolic systems, Journal of Mathematics and System Science. Vol 7, $79-87$.

[18] E. ZuAzuA (1990), Exact controllability for the semilinear wave equation, J. Math. Pures Appl, 69, 1-31. 